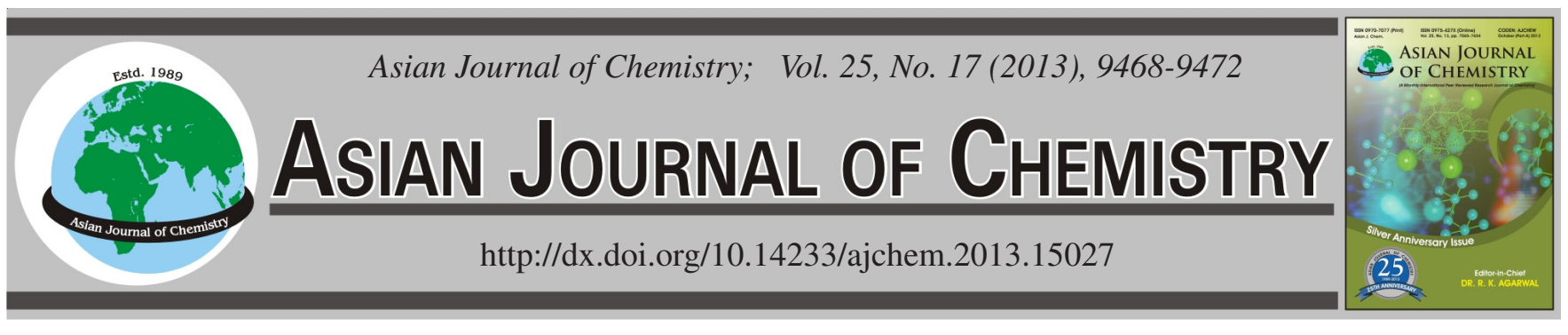

\title{
Synthesis, Spectral Analysis and Biological Evaluation of Sulfonamides Bearing Piperidine Nucleus
}

\author{
Hira Khalid ${ }^{1}$, Aziz-ur-Rehman ${ }^{1, *}$, Muhammad Athar Abbasi $^{1}$, Abdul Malik $^{2}$,

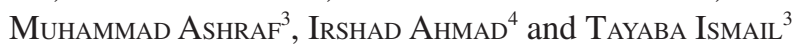

\begin{abstract}
${ }^{1}$ Department of Chemistry, Government College University, Lahore-54000, Pakistan
${ }^{2}$ HEJ Research Institute of Chemistry, International Center for Chemical and Biological Sciences, University of Karachi, Karachi-75270, Pakistan ${ }^{3}$ Department of Biochemistry and Biotechnology, The Islamia University of Bahawalpur, Bahawalpur-63100, Pakistan

${ }^{4}$ Department of Pharmacy, The Islamia University of Bahawalpur, Bahawalpur-63100, Pakistan
\end{abstract}

*Corresponding author: Tel: +92 42 111000010; Ext. 450; E-mail: azizryk@yahoo.com; hirachemist@gmail.com

\begin{abstract}
In the current study, two new series of $N$-alkyl- $N$-(piperidin-1-yl)benzenesulfonamide (3a-f) and $N$-aryl substitued 2-[(phenylsulfonyl)(piperidin-1-yl)amino]acetamide (5a-c) were synthesized and enzyme inhibiting activity was screened for all these chemical entities. The synthesis was carried out by the coupling of benzenesulfonyl chloride (a) with 1-amino piperidine (b) under dynamic $\mathrm{pH}$ control in aqueous media to afford parent compound $N$-(piperidin-1-yl)benzenesulfonamide (1). The parent compound further on treatment with different electrophiles $\mathbf{2} \mathbf{a}-\mathbf{f}$ and $\mathbf{4 a - c}$ in the presence of sodium hydride $(\mathrm{NaH})$ and $N, N$-dimethyl formamide furnished 3a-f and 3a-c derivatives, respectively. The structures of all the synthesized compounds were confirmed based on IR, EIMS and ${ }^{1} \mathrm{H}$ NMR data. The synthesized compounds were screened against acetylcholinesterase (AChE), butyrylcholinesterase (BChE) and lipoxygenase (LOX) enzymes and almost all the compounds exhibited promising activity but some of the compounds remained inactive against lipoxygenase enzymes.
\end{abstract}

Key Words: 1-Aminopiperidine, Sulfonamides, Enzyme inhibiting activity, EI-MS and ${ }^{1} \mathrm{H}$ NMR.

\section{INTRODUCTION}

The piperidine nucleus is a ubiquitous structural feature of biologically active compounds and numerous secondary metabolites, for example (S)-pipecolic acid a non-proteinogenic amino acid associated with epilepsy ${ }^{1-4}$. Piperidine bearing compounds have diverse applications in commercial and medicinal area. Piperidine and pyrrolidine ring containing compounds were evaluated for their effect on plasma glucose level ${ }^{5}$, insulin normalization and treatment of cocaine abuse ${ }^{6}$. Sulfonamides are famous for enzyme inhibition such as carbonic anhydrase, cysteine protease, HIV protease and cyclooxygenase ${ }^{7}$. Worldwide researchers are trying to synthesize new drugs with better pharmacokinetic and dynamic properties with less adverse effects.

Most of the drugs used for the treatment of Alzheimer's disease are acetylcholinesterase inhibitors. Acetylcholinesterase (AChE, EC 3.1.1.7) and butyrylcholinesterase (BChE, EC 3.1.1.8) comprise a family of enzymes which include serine hydrolases. The different specificities for substrates and inhibitors for these enzymes are due to the differences in amino acid residues of the active sites of $\mathrm{AChE}$ and $\mathrm{BChE}$. The enzyme system is responsible for the termination of acetylcholine at cholinergic synapses. These are key components of cholinergic brain synapses and neuromuscular junctions. The major function of $\mathrm{AChE}$ and $\mathrm{BChE}$ is to catalyze the hydrolysis of the neurotransmitter acetylcholine and termination of the nerve impulse in cholinergic synapses ${ }^{8,9}$. It has been found that $\mathrm{BChE}$ is present in significantly higher quantities in Alzheimer's plaques than in the normal age related nondementia of brains. Antagonists of the histamine $\mathrm{H}_{1}$ and $\mathrm{H}_{2}$ receptors have been successful as blockbuster drugs for treating allergic conditions and gastric ulcers, respectively. Cholinesterase inhibitors increase the amount of acetylcholine available for neuronal and neuromuscular transmission through their ability to reversibly or irreversibly Hence, the search for new cholinesterase inhibitors is considered an important and ongoing strategy to introduce new drug candidates for the treatment of Alzheimer's disease and other related disease ${ }^{10,11}$.

Pharmacological potential of the heterocyclic compounds motivated chemists to synthesize piperidine derivatives with improved biological activities. In continuation of our previous work on sulfonamides as possible therapeutic entrants ${ }^{12}$, here 
we report the synthesis of new sulfonamides bearing piperidine moiety with enzyme inhibition activity.

\section{EXPERIMENTAL}

Chemicals were purchased from Sigma Aldrich and Alfa Aesar (Germany). By using open capillary tube method melting points were taken on Griffin and George melting point apparatus. Melting points were uncorrected. By using thin layer chromatography and $n$-hexane and ethyl acetate as mobile phase purity of the synthesized compounds was detected at $364 \mathrm{~nm}$. By using KBr, IR peaks were recorded on a Jasco-320-A spectrophotometer. ${ }^{1} \mathrm{H}$ NMR signals were recorded at $300 \mathrm{MHz}$ in $\mathrm{CD}_{3} \mathrm{OD}$ using Bruker spectrometers, chemical shift values are mentioned in ppm unit. EIMS signals are recorded by utilizing a JMS-HX-110 spectrometer.

Procedure for synthesis of $N$-(piperidin-1-yl)benzenesulfonamide in aqueous medium (1): 1-Amino piperidine (a) $(2.6 \mathrm{~mL} ; 10 \mathrm{mmol})$ was suspended in $50 \mathrm{~mL}$ water and the $\mathrm{pH}$ was maintained at 9 by adding basic aqueous solution of a $\mathrm{Na}_{2} \mathrm{CO}_{3}$ at $0-5{ }^{\circ} \mathrm{C}$. Then, benzenesulfonyl chloride (b) (2.9 $\mathrm{mL} ; 10.0 \mathrm{mmol}$ ) was added in the reaction mass slowly over 10-15 min. After completion of the addition, the temperature of the reaction mixture was allowed to rise slowly to room temperature. The reaction mixture was stirred and monitored with TLC for the completion of reaction. Then conc. $\mathrm{HCl}$ (around $2 \mathrm{~mL}$ ) was added slowly to adjust the $\mathrm{pH}$ to 2 . The reaction mass was cooled to room temperature, filtered and the solid washed with distilled water to afford the title compound 1 on drying.

General procedure for synthesis of compounds 3a-f: To a solution of compound $\mathbf{1}(0.2 \mathrm{~g}, 8.33 \mathrm{mmol})$ in $N, N$-dimethyl formamide $(5 \mathrm{~mL})$ was added sodium hydride $(0.01 \mathrm{~g}, 0.40$ $\mathrm{mmol}$ ) at $0-5^{\circ} \mathrm{C}$. After completion of the addition, the temperature of the reaction mass was raised to room temperature and stirred for $15 \mathrm{~min}$. The corresponding alkyl halide $(8.33$ $\mathrm{mmol}$ ) was added into the reaction mixture and stirred for 30$40 \mathrm{~min}$. The reaction mass was then monitored by TLC. After complete conversion, the reaction mass was cooled to room temperature and quenched with cold water $(200 \mathrm{~mL})$. The obtained solid was filtered, washed with water and dried to yield the corresponding $N$-alkyl derivatives 3a-f.

The corresponding alkyl halides used for the reactions were ethyl iodide, allyl iodide, benzyl chloride, 4-bromobenzyl bromide, 2-phenyl ethyl iodide and 3-phenyl propyl iodide.

General procedure for synthesis of compounds 4a-c: The calculated amount of substituted aromatic amines/aromatic alkyl amines (11 mmol) was taken in an iodine flask containing $10 \mathrm{~mL}$ of distilled water and $5 \% \mathrm{Na}_{2} \mathrm{CO}_{3}$ solution was added to adjust the $\mathrm{pH}$ 8.0-9.0. After adjusting the $\mathrm{pH}$, the temperature of the reaction mass was maintained at $0-5^{\circ} \mathrm{C}$ and stirred for $0.5 \mathrm{~h}$. The bromoacetyl bromide $(1 \mathrm{~mL} ; 11 \mathrm{mmol}$ ) was further poured drop wise in the reaction mass in $2-5$ min at $0-5{ }^{\circ} \mathrm{C}$. After completion of the addition, the iodine flask was vigorously shaken (manually) till the solid precipitates formed and the temperature of the reaction was allowed to rise slowly to room temperature. The solid precipitate was further stirred for $45 \mathrm{~min}$. The progress of reaction completion was monitored by TLC ( $n$-hexane:ethyl acetate: 70:30). After complete conversion, the obtained solid was filtered, washed with distilled water and dried to yield the corresponding electrophile $N$-alkylsubstituted-2-bromoacetamide 4a-c.

General procedure for synthesis of $\mathrm{N}$-substituted-2[(phenylsulfonyl) (piperidin-1-yl)amino] acetamide (5a-c): To a solution of compound $1(0.1 \mathrm{~g}, 0.40 \mathrm{mmol})$ in DMF $(5 \mathrm{~mL})$ was added sodium hydride $(0.01 \mathrm{~g}, 0.40 \mathrm{mmol})$ in small portions over $2-5 \mathrm{~min}$ at $0-5^{\circ} \mathrm{C}$. After addition, the temperature was maintained at room temperature and stirred for $15 \mathrm{~min}$. The corresponding $N$-substituted aryl/alkyl-2-bromoacetamide $(0.09 \mathrm{~g}, 0.40 \mathrm{mmol})$ was added into the reaction mixture slowly and stirred for 10-15 min. The reaction mixture was then heated to $50{ }^{\circ} \mathrm{C}$ and stirred at this temperature $30-40 \mathrm{~min}$; progress was monitored by TLC. After completion of reaction, mixture was cooled to room temperature and quenched with cold water $(50 \mathrm{~mL})$. The acquired precipitation was filtered, washed with water and dried to acquire the resultant derivatives. In some cases, the solid precipitation was not formed in the flask then compound was extracted through solvent extraction method by chloroform/ethyl acetate to yield the corresponding $N$ substituted 2-[(phenylsulfonyl)(piperidin-1-yl)amino]acetamide (5a-c) derivatives.

\section{Spectral characterization of synthesized compounds}

$N$-(Piperidin-1-yl)benzenesulfonamide (1): IR (KBr, $\left.V_{\max }, \mathrm{cm}^{-1}\right): 3430(\mathrm{~N}-\mathrm{H}$ stretching), 3024 (C-H stretching of aromatic ring), $1546(\mathrm{C}=\mathrm{C}$ stretching of aromatic ring), 1341 (-SO ${ }_{2}$-stretching), ${ }^{1} \mathrm{H} \mathrm{NMR}$ (300 MHz, $\left.\mathrm{CD}_{3} \mathrm{OD}, \delta / \mathrm{ppm}\right): 7.90$ (dd, $J=8.7,1.5 \mathrm{~Hz}, 2 \mathrm{H}, \mathrm{H}-2^{\prime}$ and $\left.\mathrm{H}-6 '\right), 7.65$ (m, 2H, H-3' and H-5'), 7.52 (m, 1H, H-4'), 2.96 (t, $J=5.4 \mathrm{~Hz}, 2 \mathrm{H}, \mathrm{H}_{\mathrm{eq}}-2$ and $\left.\mathrm{H}_{\mathrm{eq}}-6\right), 2.47\left(\mathrm{t}, J=5.4 \mathrm{~Hz}, 2 \mathrm{H}, \mathrm{H}_{\mathrm{ax}}-2\right.$ and $\left.\mathrm{H}_{\mathrm{ax}}-6\right), 1.61(\mathrm{~m}, 2 \mathrm{H}$, $\left.\mathrm{CH}_{2}-4\right), 1.44$ (m, 4H, $\mathrm{CH}_{2}-3$ and $\mathrm{CH}_{2}-5$ ). EIMS m/z: 240 (24 $\%)[\mathrm{M}]^{+}, 176(37 \%), 156(54 \%), 141(100 \%)$.

$\mathrm{N}$-Ethyl- $\boldsymbol{N}$-(piperidin-1-yl)benzenesulfonamide (3a): IR (KBr, $\left.v_{\max }, \mathrm{cm}^{-1}\right): 2915$ (- $\mathrm{CH}_{2}$-stretching), 3029 (C-H stretching of aromatic ring), $1541(\mathrm{C}=\mathrm{C}$ stretching of aromatic ring), 1337 (-SO $\mathrm{SO}_{2}$-stretching), ${ }^{1} \mathrm{H} \mathrm{NMR}\left(300 \mathrm{MHz}, \mathrm{CD}_{3} \mathrm{OD}\right.$, S/ppm): 7.90 (dd, $J=8.4,1.2 \mathrm{~Hz}, 2 \mathrm{H}, \mathrm{H}-2^{\prime}$ and H-6'), 7.65 (m, $2 \mathrm{H}, \mathrm{H}-3^{\prime}$ and $\left.\mathrm{H}-5^{\prime}\right), 7.52\left(\mathrm{~m}, 1 \mathrm{H}, \mathrm{H}-4^{\prime}\right), 2.95$ (t, $J=5.4 \mathrm{~Hz}, 2 \mathrm{H}$, $\mathrm{H}_{\mathrm{eq}}-2$ and $\left.\mathrm{H}_{\mathrm{eq}}-6\right), 2.47\left(\mathrm{t}, J=5.4 \mathrm{~Hz}, 2 \mathrm{H}, \mathrm{H}_{\mathrm{ax}}-2\right.$ and $\left.\mathrm{H}_{\mathrm{ax}}-6\right)$, 1.61 (m, 2H, $\left.\mathrm{CH}_{2}-4\right), 1.44$ ( $\mathrm{m}, 4 \mathrm{H}, \mathrm{CH}_{2}-3$ and $\left.\mathrm{CH}_{2}-5\right), 3.20$ (q, $\left.J=7.5 \mathrm{~Hz}, 2 \mathrm{H}, \mathrm{CH}_{2}-1 "\right), 1.20$ (t, $\left.J=7.5,3 \mathrm{H}, \mathrm{CH}_{3}-2 "\right)$. EIMS $\mathrm{m} / \mathrm{z}: 268(19 \%)[\mathrm{M}]^{+}, 204(34 \%), 184(47 \%), 127(100 \%)$.

$\mathrm{N}$-Allyl- $\mathrm{N}$-(piperidin-1-yl)benzenesulfonamide (3b): IR $\left(\mathrm{KBr}, v_{\max }, \mathrm{cm}^{-1}\right): 2919\left(-\mathrm{CH}_{2}\right.$-stretching), 3035 (C-H stretching of aromatic ring), 1543 (C=C stretching of aromatic ring), 1335 (-SO ${ }_{2}$-stretching), ${ }^{1} \mathrm{H} \mathrm{NMR}$ (300 MHz, $\left.\mathrm{CD}_{3} \mathrm{OD}, \delta / \mathrm{ppm}\right): 7.90$ (dd, $J=8.7,1.5 \mathrm{~Hz}, 2 \mathrm{H}, \mathrm{H}-2^{\prime}$ and H-6'), 7.65 (m, 2H, H-3' and H-5'), 7.52 (m, 1H, H-4'), 5.78 (m, 1H, H-2"), 5.03 (dd, $J=1.6$, $\left.17.3 \mathrm{~Hz}, 1 \mathrm{H}, \mathrm{H}_{\mathrm{b}}-3 "\right), 4.96$ (dd, $\left.J=1.2,10.0 \mathrm{~Hz}, 1 \mathrm{H}, \mathrm{H}_{\mathrm{a}}-3^{\prime \prime}\right)$, 4.47 (s, 2H, $\left.\mathrm{CH}_{2}-1 "\right), 3.10$ (t, $J=5.4 \mathrm{~Hz}, 2 \mathrm{H}, \mathrm{H}_{\mathrm{eq}}-2$ and $\left.\mathrm{H}_{\mathrm{eq}}-6\right)$, $2.47\left(\mathrm{t}, J=5.4 \mathrm{~Hz}, 2 \mathrm{H}, \mathrm{H}_{\mathrm{ax}}-2\right.$ and $\left.\mathrm{H}_{\mathrm{ax}}-6\right), 1.61\left(\mathrm{~m}, 2 \mathrm{H}, \mathrm{CH}_{2}-4\right)$, $1.44\left(\mathrm{~m}, 4 \mathrm{H}, \mathrm{CH}_{2}-3\right.$ and $\left.\mathrm{CH}_{2}-5\right)$. EIMS $m / z: 280(15 \%)[\mathrm{M}]^{+}$, $216(35 \%), 239(22 \%), 196(55 \%), 139(100 \%)$.

$\boldsymbol{N}$-Benzyl- $\boldsymbol{N}$-(piperidin-1-yl)benzenesulfonamide (3c): IR ( $\left.\mathrm{KBr}, \mathrm{v}_{\max }, \mathrm{cm}^{-1}\right): 2919$ (- $\mathrm{CH}_{2}$-stretching), 3035 (C-H stretching of aromatic ring), $1543(\mathrm{C}=\mathrm{C}$ stretching of aromatic ring), 1335 (-SO $\mathrm{SO}_{2}$-stretching), ${ }^{1} \mathrm{H} \mathrm{NMR}\left(300 \mathrm{MHz}, \mathrm{CD}_{3} \mathrm{OD}\right.$,

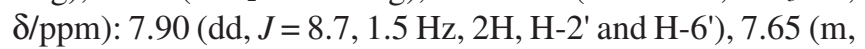


$2 \mathrm{H}, \mathrm{H}-3^{\prime}$ and $\left.\mathrm{H}-5^{\prime}\right), 7.52$ (m, 1H, H-4'), 7.48 (m, 2H, H-3" and H-5"), 7.25 (m, 1H, H-4"), 7.11 (d, $J=7.00 \mathrm{~Hz}, 2 \mathrm{H}, \mathrm{H}-2 "$ and H-6"), 4.47 (s, 2H, $\left.\mathrm{CH}_{2}-7 "\right), 3.10$ (t, $J=5.4 \mathrm{~Hz}, 2 \mathrm{H}, \mathrm{H}_{\mathrm{eq}}-2$ and $\left.\mathrm{H}_{\mathrm{eq}}-6\right), 2.47\left(\mathrm{t}, J=5.4 \mathrm{~Hz}, 2 \mathrm{H}, \mathrm{H}_{\mathrm{ax}}-2\right.$ and $\left.\mathrm{H}_{\mathrm{ax}}-6\right), 1.61(\mathrm{~m}, 2 \mathrm{H}$, $\left.\mathrm{CH}_{2}-4\right), 1.44$ (m, 4H, $\mathrm{CH}_{2}-3$ and $\mathrm{CH}_{2}-5$ ), EIMS m/z: 330 (23 $\%)[\mathrm{M}]^{+}, 266(44 \%), 239(25 \%), 246(65 \%), 91(100 \%)$.

$\boldsymbol{N}$-(4-Bromobenzyl)- $\boldsymbol{N}$-(piperidin-1-yl)benzenesulfonamide (3d): IR ( $\left.\mathrm{KBr}, \nu_{\max }, \mathrm{cm}^{-1}\right)$ : $2923\left(-\mathrm{CH}_{2}\right.$-stretching), 3029 (C-H stretching of aromatic ring), $1549(\mathrm{C}=\mathrm{C}$ stretching of aromatic ring), 1329 (-SO $\mathrm{SO}_{2}$-stretching), ${ }^{1} \mathrm{H} \mathrm{NMR}(300 \mathrm{MHz}$, $\left.\mathrm{CD}_{3} \mathrm{OD}, \delta / \mathrm{ppm}\right): 7.68\left(\mathrm{dd}, J=8.7,1.5 \mathrm{~Hz}, 2 \mathrm{H}, \mathrm{H}-2^{\prime}\right.$ and $\left.\mathrm{H}-66^{\prime}\right)$, $7.54\left(\mathrm{~m}, 2 \mathrm{H}, \mathrm{H}-3^{\prime}\right.$ and $\left.\mathrm{H}-5^{\prime}\right), 7.46\left(\mathrm{~d}, J=8.0 \mathrm{~Hz}, 2 \mathrm{H}, \mathrm{H}-3^{\prime \prime}\right.$ and H-5"), 7.39 (m, 1H, H-4'), 7.02 (d, $J=8.0 \mathrm{~Hz}, 2 \mathrm{H}, \mathrm{H}-2^{\prime \prime}$ and H-6"), 4.47 (s, 2H, $\mathrm{CH}_{2}-7$ "), 3.14 (t, $J=5.4 \mathrm{~Hz}, 2 \mathrm{H}, \mathrm{H}_{\mathrm{eq}}-2$ and $\left.\mathrm{H}_{\mathrm{eq}}-6\right), 2.47\left(\mathrm{t}, J=5.4 \mathrm{~Hz}, 2 \mathrm{H}, \mathrm{H}_{\mathrm{ax}}-2\right.$ and $\left.\mathrm{H}_{\mathrm{ax}}-6\right), 1.71(\mathrm{~m}, 2 \mathrm{H}$, $\left.\mathrm{CH}_{2}-4\right), 1.56$ (m, 4H, $\mathrm{CH}_{2}-3$ and $\left.\mathrm{CH}_{2}-5\right)$. EIMS m/z: 409 (13 $\%)[\mathrm{M}]^{+}, 345$ (29\%), 325 (33\%), 268 (49\%), $171(100 \%)$.

$\boldsymbol{N}$-[(2-Phenylethyl)- $\boldsymbol{N}$-(piperidin-1-yl)benzenesulfonamide (3e): IR (KBr, $\left.\mathrm{v}_{\max }, \mathrm{cm}^{-1}\right): 2921\left(-\mathrm{CH}_{2}\right.$-stretching), 3034 (C-H stretching of aromatic ring), $1541(\mathrm{C}=\mathrm{C}$ stretching of aromatic ring), 1332 (-SO $\mathrm{SO}_{2}$-stretching), ${ }^{1} \mathrm{H} \mathrm{NMR}(300 \mathrm{MHz}$, $\mathrm{CD}_{3} \mathrm{OD}, \delta / \mathrm{ppm}$ ): 7.86 (dd, 2H, $J=7.2,1.5 \mathrm{~Hz}, \mathrm{H}-2^{\prime}$ and $\mathrm{H}-6{ }^{\prime}$ ), 7.69 (m, 2H, H-3' and H-5'), $7.61\left(\mathrm{~m}, 1 \mathrm{H}, \mathrm{H}-4{ }^{\prime}\right), 7.17-7.21$ (m, 5H, H-2"' to H-6"'), 3.55 (t, $J=5.4 \mathrm{~Hz}, 2 \mathrm{H}, \mathrm{H}_{\mathrm{eq}}-2$ and $\mathrm{H}_{\mathrm{eq}}{ }^{-}$ 6), 3.15 ( t $, J=5.4 \mathrm{~Hz}, 2 \mathrm{H}, \mathrm{CH}_{2}-8$ "), 2.55 (t, $J=5.4 \mathrm{~Hz}, 2 \mathrm{H}$, $\mathrm{H}_{\mathrm{ax}}-2$ and $\left.\mathrm{H}_{\mathrm{ax}}-6\right), 2.66\left(\mathrm{t}, J=5.4 \mathrm{~Hz}, 2 \mathrm{H}, \mathrm{CH}_{2}-7 "\right), 1.61(\mathrm{~m}$, $\left.2 \mathrm{H}, \mathrm{CH}_{2}-4\right), 1.44\left(\mathrm{~m}, 4 \mathrm{H}, \mathrm{CH}_{2}-3\right.$ and $\left.\mathrm{CH}_{2}-5\right)$. EIMS $m / z: 344$ (16\%) [M] $]^{+}, 280(35 \%), 239(54 \%), 260(74 \%), 105(100 \%)$.

$N$-(3-Phenylpropyl)- $N$-(piperidin-1-yl)benzenesulfonamide (3f): IR (KBr, $\left.v_{\max }, \mathrm{cm}^{-1}\right)$ : $2917\left(-\mathrm{CH}_{2}\right.$ stretching), 3037 (C-H stretching of aromatic ring), $1543(\mathrm{C}=\mathrm{C}$ stretching of aromatic ring), 1323 (- $\mathrm{SO}_{2}$-stretching), ${ }^{1} \mathrm{H} \mathrm{NMR}(300 \mathrm{MHz}$, $\left.\mathrm{CD}_{3} \mathrm{OD}, \delta / \mathrm{ppm}\right): 7.86$ (dd, $J=7.2,1.5 \mathrm{~Hz}, 2 \mathrm{H}, \mathrm{H}-2^{\prime}$ and $\left.\mathrm{H}-6{ }^{\prime}\right)$, 7.69 (m, 2H, H-3' and H-5'), 7.61 (m, 1H, H-4'), 7.17 (m, 5H, H-2" to H-6"), 3.55 (t, $J=5.4 \mathrm{~Hz}, 2 \mathrm{H}, \mathrm{H}_{\mathrm{eq}}-2$ and $\left.\mathrm{H}_{\mathrm{eq}}-6\right), 3.15$ (t, $\left.J=5.4 \mathrm{~Hz}, 2 \mathrm{H}, \mathrm{CH}_{2}-9 "\right), 2.55\left(\mathrm{t}, J=5.4 \mathrm{~Hz}, 2 \mathrm{H}, \mathrm{H}_{\mathrm{ax}}-2\right.$ and $\left.\mathrm{H}_{\mathrm{ax}}-6\right), 2.66$ (t, J = 5.4 Hz, 2H, $\left.\mathrm{CH}_{2}-7^{\prime \prime}\right), 1.93$ (m, 2H, $\mathrm{CH}_{2^{-}}$ 8"), 1.61 (m, 2H, $\left.\mathrm{CH}_{2}-4\right), 1.44\left(\mathrm{~m}, 4 \mathrm{H}, \mathrm{CH}_{2}-3\right.$ and $\left.\mathrm{CH}_{2}-5\right)$. EIMS m/z: $358(16 \%)\left[\mathrm{M}^{+}, 294(35 \%), 274\right.$ (54\%), 239 (74\%), $141(100 \%)$.

$\mathrm{N}$-(2-Methylphenyl)-2-[(phenylsulfonyl)(piperidin-1yl)amino]acetamide (5a): IR $\left(\mathrm{KBr}, \mathrm{v}_{\max }, \mathrm{cm}^{-1}\right): 3450(\mathrm{~N}-\mathrm{H}$ stretching), 3015 (C-H stretching of aromatic ring), 2912 ($\mathrm{CH}_{2}$-stretching), $1526(\mathrm{C}=\mathrm{C}$ stretching of aromatic ring), 1337 (-SO ${ }_{2}$-stretching), ${ }^{1} \mathrm{H}$ NMR (300 MHz, $\left.\mathrm{CD}_{3} \mathrm{OD}, \delta / \mathrm{ppm}\right): 11.09$ (s, 1H, N-H), 7.98 (dd, $J=8.7,1.5 \mathrm{~Hz}, 2 \mathrm{H}, \mathrm{H}-2^{\prime}$ and $\left.\mathrm{H}-6{ }^{\prime}\right)$, $7.73\left(\mathrm{~m}, 2 \mathrm{H}, \mathrm{H}-3^{\prime}\right.$ and $\left.\mathrm{H}-5^{\prime}\right), 7.61$ (m, 1H, H-4'), 7.11-7.28 (m, 4H, H-3"' to H-6"'), 4.04 (s, 2H, $\mathrm{CH}_{2}-2$ "), 3.14 (t, $J=5.4$ $\mathrm{Hz}, 2 \mathrm{H}, \mathrm{H}_{\mathrm{eq}}-2$ and $\left.\mathrm{H}_{\mathrm{eq}}-6\right), 2.47\left(\mathrm{t}, J=5.4 \mathrm{~Hz}, 2 \mathrm{H}, \mathrm{H}_{\mathrm{ax}}-2\right.$ and $\left.\mathrm{H}_{\mathrm{ax}}-6\right), 2.19$ (s, 3H, $\mathrm{CH}_{3}-2$ "'), 1.71 (m, 2H, $\left.\mathrm{CH}_{2}-4\right), 1.56$ (m, $4 \mathrm{H}, \mathrm{CH}_{2}-3$ and $\left.\mathrm{CH}_{2}-5\right)$. EIMS m/z: 387 (16\%) [M] $]^{+}, 323$ (35 $\%), 372(54 \%), 281$ (74\%), 105 (100\%).

$\mathrm{N}$-(2-Methoxyphenyl)-2-[(phenylsulfonyl)(piperidin-1yl)amino]acetamide (5b): IR ( KBr, $\left.v_{\max }, \mathrm{cm}^{-1}\right): 3443(\mathrm{~N}-\mathrm{H}$ stretching), 3019 (C-H stretching of aromatic ring), 2923 ( $-\mathrm{CH}_{2}$-stretching), 1531 ( $\mathrm{C}=\mathrm{C}$ stretching of aromatic ring), 1325 (- $\mathrm{SO}_{2}$-stretching), ${ }^{1} \mathrm{H} \mathrm{NMR}\left(300 \mathrm{MHz}, \mathrm{CD}_{3} \mathrm{OD}, \delta / \mathrm{ppm}\right)$ : 11.02 (s, 1H, N-H), 7.74 (dd, $J=6.6,1.8$ Hz, 2H, H-2', H-6'), $7.61\left(\mathrm{~m}, 2 \mathrm{H}, \mathrm{H}-3^{\prime}\right.$ and H-5'), 6.95-6.99 (m, 4H, H-3'" to H6"'), 6.80 (m, 1H, H-4'), 3.90 (s, 3H, $\left.\mathrm{CH}_{3}-2^{\prime \prime \prime}\right), 4.09$ (s, 2H, $\mathrm{CH}_{2}-2$ "), 2.96 (t, $J=5.4 \mathrm{~Hz}, 2 \mathrm{H}, \mathrm{H}_{\mathrm{eq}}-2$ and $\left.\mathrm{H}_{\mathrm{eq}}-6\right), 2.47$ (t, $J=$ $5.4 \mathrm{~Hz}, 2 \mathrm{H}, \mathrm{H}_{\mathrm{ax}}-2$ and $\left.\mathrm{H}_{\mathrm{ax}}-6\right), 1.71\left(\mathrm{~m}, 2 \mathrm{H}, \mathrm{CH}_{2}-4\right), 1.61$ (m, $4 \mathrm{H}, \mathrm{CH}_{2}-3$ and $\left.\mathrm{CH}_{2}-5\right)$. EIMS $m / z: 401$ (13\%) [M] $]^{+}, 372$ (43\%), 281 (49\%), 239 (71\%), $141(100 \%)$.

$\mathrm{N}$-(3-Methoxyphenyl)-2-[(phenylsulfonyl)(piperidin-1yl)amino]acetamide (5c): IR $\left(\mathrm{KBr}, v_{\max }, \mathrm{cm}^{-1}\right): 3440(\mathrm{~N}-\mathrm{H}$ stretching), 3021 (C-H stretching of aromatic ring), 2920 (- $\mathrm{CH}_{2}$-stretching), 1529 ( $\mathrm{C}=\mathrm{C}$ stretching of aromatic ring), 1323 (-SO $\mathrm{SO}_{2}$-stretching), ${ }^{1} \mathrm{H} \mathrm{NMR}\left(300 \mathrm{MHz}, \mathrm{CD}_{3} \mathrm{OD}, \delta / \mathrm{ppm}\right)$ : 11.13 (s, 1H, N-H), 7.72 (dd, $J=6.6,1.8 \mathrm{~Hz}, 2 \mathrm{H}, \mathrm{H}-2^{\prime}$ and H-6'), 7.63 (m, 2H, H-3' and H-5'), 7.23 (m, 1H, H-4'), 7.11 (t, $\left.J=8.1 \mathrm{~Hz}, 1 \mathrm{H}, \mathrm{H}-5^{\prime \prime}\right), 7.09$ (dd, $\left.J=8.1,1.2 \mathrm{~Hz}, 1 \mathrm{H}, \mathrm{H}-66^{\prime \prime}\right)$, 6.76 (d, $\left.J=1.5 \mathrm{~Hz}, 1 \mathrm{H}, \mathrm{H}-22^{\prime \prime}\right), 6.52$ (dd, $J=8.1,1.2 \mathrm{~Hz}, 1 \mathrm{H}$, H-4"'), 4.15 (s, 2H, $\mathrm{CH}_{2}-2$ "), 3.92 (s, 3H, $\mathrm{CH}_{3}-3$ "?), 2.92 (t, $J$ $=5.4 \mathrm{~Hz}, 2 \mathrm{H}, \mathrm{H}_{\mathrm{eq}}-2$ and $\left.\mathrm{H}_{\mathrm{eq}}-6\right), 2.43\left(\mathrm{t}, J=5.4 \mathrm{~Hz}, 2 \mathrm{H}, \mathrm{H}_{\mathrm{ax}}-2\right.$ and $\left.\mathrm{H}_{\mathrm{ax}}-6\right), 1.73\left(\mathrm{~m}, 2 \mathrm{H}, \mathrm{CH}_{2}-4\right), 1.60\left(\mathrm{~m}, 4 \mathrm{H}, \mathrm{CH}_{2}-3\right.$ and $\mathrm{CH}_{2-}$ 5). EIMS $m / z: 401(10 \%)[\mathrm{M}]+, 372(32 \%), 281(54 \%), 239$ (61\%), $141(100 \%)$.

\section{Enzyme inhibition essays}

Cholinesterase assay: The $\mathrm{AChE}$ and $\mathrm{BChE}$ inhibition activity was performed according to the reported method ${ }^{13}$ with slight modifications. Total volume of the reaction mixture was $100 \mu \mathrm{L}$. It contained $60 \mu \mathrm{L} \mathrm{Na}_{2} \mathrm{HPO}_{4}$ buffer with concentration of $50 \mathrm{mM}$ and $\mathrm{pH}$ 7.7. Ten $\mu \mathrm{L}$ test compound $\left(0.5 \mathrm{mM}\right.$ well $\left.{ }^{-1}\right)$ was added, followed by the addition of $10 \mu \mathrm{L}$ ( 0.005 unit well- 1$)$ enzyme (Sigma, USA). The contents were mixed and pre-read at $405 \mathrm{~nm}$. Then contents were pre-incubated for $10 \mathrm{~min}$ at $37^{\circ} \mathrm{C}$. The reaction was initiated by the addition of $10 \mu \mathrm{L}$ of $0.5 \mathrm{mM}$ well ${ }^{-1}$ substrate (acetylthiocholine iodide or butyrylthiocholine chloride), followed by the addition of $10 \mu \mathrm{L}$ DTNB $(0.5 \mathrm{mM}$ well $\left.{ }^{-1}\right)$. After $15 \mathrm{~min}$ of incubation at $37^{\circ} \mathrm{C}$ absorbance was measured at $405 \mathrm{~nm}$ using 96-well plate reader Synergy HT, Biotek, USA. All experiments were carried out with their respective controls in triplicate. Eserine $\left(0.5 \mathrm{mM}\right.$ well $\left.{ }^{-1}\right)$ was used as a positive control. The percent inhibition was calculated by the help of following equation:

$$
\text { Inhibition }(\%)=\frac{\text { Control }- \text { Test }}{\text { Control }} \times 100
$$

$\mathrm{IC}_{50}$ values were calculated using EZ-Fit Enzyme kinetics software (Perrella Scientific Inc. Amherst, USA).

Lipoxygenase assay: Lipoxygenase (LOX) activity was assayed according to the reported method ${ }^{14-16}$ with slight modifications. A total volume of $200 \mu \mathrm{L}$ lipoxygenase assay mixture contained $150 \mu \mathrm{L}$ sodium phosphate buffer $(100 \mathrm{mM}$, $\mathrm{pH}$ 8.0), $10 \mu \mathrm{L}$ test compound and $15 \mu \mathrm{L}$ purified lipoxygenase enzyme (600 units well ${ }^{-1}$, Sigma Inc.). The contents were mixed and pre-read at $234 \mathrm{~nm}$ and preincubated for $10 \mathrm{~min}$ at $25^{\circ} \mathrm{C}$. The reaction was initiated by addition of $25 \mu \mathrm{L}$ substrate solution. The change in absorbance was observed after 6 min at $234 \mathrm{~nm}$ using 96-well plate reader Synergy HT, Biotek, USA. All reactions were performed in triplicates. The positive and negative controls were included in the assay. Baicalin $\left(0.5 \mathrm{mM}\right.$ well $\left.{ }^{-1}\right)$ was used as a positive control. The percentage inhibition and $\mathrm{IC}_{50}$ values were calculated as mentioned above. 
Statistical analysis: All the measurements were done in triplicate and statistical analysis was performed by Microsoft Excel 2003. Results are presented as mean \pm sem.

\section{RESULTS AND DISCUSSION}

In the undertaken research, new series of heterocyclic compounds containing piperidine nucleus was synthesized as described in Scheme-I. The structures of all these compounds were characterized by spectral data. The parent compound $N$-(piperidin-1-yl)benzenesulfonamide (1), was prepared by the coupling of benzenesulfonyl chloride (a) with 1-amino piperidine (b) under the dynamic $\mathrm{pH}$ control of basic aqueous media $^{17}$. Further, the reaction of parent compound with different electrophiles yielded a series of $N$-alkyl substituted- $N$ (piperidin-1yl)benzenesulfonamide (3a-f) and $N$-aryl substitued 2-[(phenylsulfonyl)(piperidin-1-yl)amino]acetamide (5a-c) derivatives as represented in Scheme-I. Synthesis of all the derivatives 3a-f and 5a-c was performed in DMF using sodium hydride which acts as a base. Complete conversion was achieved within 30-70 min by stirring at room temperature. The products were isolated by adding cold water in the reaction mixture and filtering off the precipitated solid. In some cases, compound was taken out through solvent extraction method by chloroform/ ethyl acetate. Parent compound 1 was synthesized as light yellow powder. The molecular formula $\mathrm{C}_{18} \mathrm{H}_{17} \mathrm{~N}_{3} \mathrm{O}_{3} \mathrm{~S}$ was established by EI-MS and counting the number of protons in the ${ }^{1} \mathrm{H}$ NMR spectrum showing molecular ion peak at $m / z 240$. The IR spectrum showed absorption bands at $3430,3024,1546$ and $1341 \mathrm{~cm}^{-1}$ which were assigned to, $\mathrm{SO}_{2}-\mathrm{N}-\mathrm{H}$ (stretching of sulfonamide), $\mathrm{C}-\mathrm{H}$ (aromatic stretching), $\mathrm{C}=\mathrm{C}$ (stretching of aromatic ring) and $-\mathrm{SO}_{2}$ (stretching of sulfonyl group), respectively. The EI-MS gave characteristic peaks at $\mathrm{m} / \mathrm{z}, 176$ and 156 which were attributed to the loss of $\mathrm{SO}_{2}$ (sulfonyl) and 1-piperidinyl groups, respectively. In the aromatic region of the ${ }^{1} \mathrm{H}$ NMR spectrum signals appeared at $\delta 7.90\left(\mathrm{dd}, J=8.7,1.5 \mathrm{~Hz}, 2 \mathrm{H}, \mathrm{H}-2^{\prime}\right.$ and $\left.\mathrm{H}-6{ }^{\prime}\right), 7.65$ (m, 2H, $\mathrm{H}-3^{\prime}$ and $\left.\mathrm{H}-5^{\prime}\right)$ and $7.52\left(\mathrm{~m}, 1 \mathrm{H}, \mathrm{H}-4^{\prime}\right)$ which were assigned to the mono substituted benzenesulfonyl ring. In the aliphatic region of the ${ }^{1} \mathrm{H}$ NMR spectrum, signals appeared at $2.96(\mathrm{t}$, $J=5.4 \mathrm{~Hz}, 2 \mathrm{H}, \mathrm{H}_{\mathrm{ax}}-2$ and $\left.\mathrm{H}_{\mathrm{ax}}-6\right), 2.47(\mathrm{t}, J=5.4 \mathrm{~Hz}, 2 \mathrm{H}$, $\mathrm{H}_{\mathrm{eq}}-2$ and $\left.\mathrm{H}_{\mathrm{eq}}-6\right), 1.61\left(\mathrm{~m}, 2 \mathrm{H}, \mathrm{CH}_{2}-4\right)$ and 1.44 (m, 4H, $\mathrm{CH}_{2}-3$ and $\mathrm{CH}_{2}-5$ ) which indicated the presence of piperidine nucleus in the molecule. On the basis of above cumulative evidences, the structure of $\mathbf{1}$ was assigned as $N$-(piperidin-1-yl)benzenesulfonamide. Similarly, the structure of other compounds was characterized by ${ }^{1} \mathrm{H}$ NMR, IR and mass spectral data as described in experimental section. The physical data of all the synthesized compounds has been shown in Table-1.

Enzyme inhibition activity: The screening of these synthesized compounds against acetylcholinesterase (AChE), butyrylcholinesterase (BChE) and lipoxygenase (LOX) enzymes revealed that these molecules exhibited good inhibitory potential against acetylcholinesterase and butyrylcholinesterase as it was evident from their $\mathrm{IC}_{50}$ values. It is obvious from Table-2 that compounds $N$-allyl- $N$-(piperidin-1-yl)benzenesulfonamide (3b) and $N$-benzyl- $N$-(piperidin-1-yl)benzenesulfonamide (3c) were showed promising inhibitory potential against butyrylcholinesterase enzyme having $\mathrm{IC}_{50}$ value of 4.4 \pm 0.03 and $4.21 \pm 0.11 \mu \mathrm{mol} / \mathrm{L}$, respectively, relative to eserine, a reference standard with $\mathrm{IC}_{50}$ value of $0.85 \pm 0.001 \mu \mathrm{mol} / \mathrm{L}$, probably due to the $N$-substitution of allyl and benzyl groups, respectively in these molecules. Similarly, from a series of $N$ -

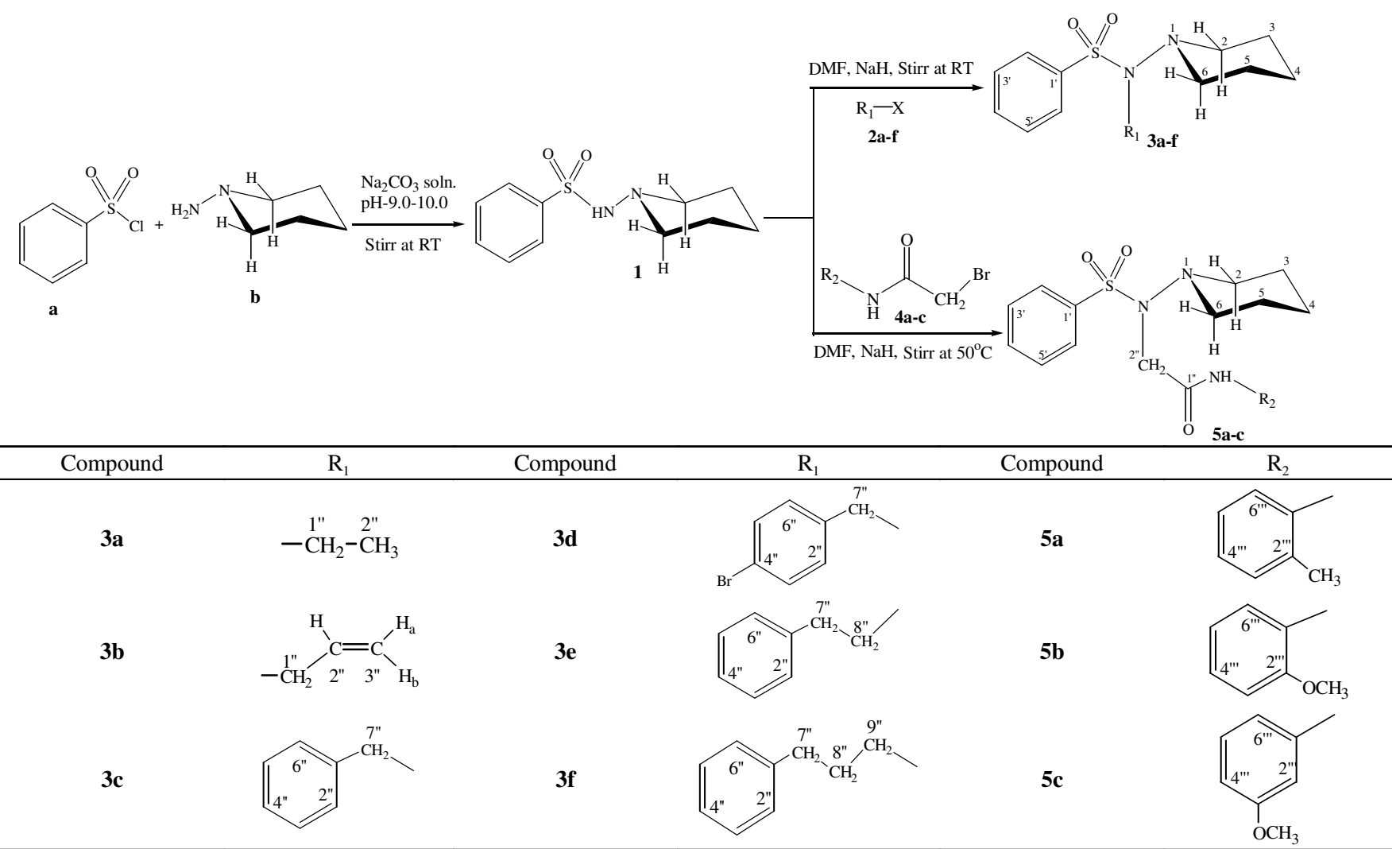

Scheme-I: Outline for the synthesis of sulfonamides bearing piperidine nucleus 
TABLE-1

PHYSICAL DATA OF THE SYNTHESIZED COMPOUNDS

\begin{tabular}{cccc}
\hline Samples & Appearance & m.p. $\left({ }^{\circ} \mathrm{C}\right)$ & Molecular formula \\
\hline $\mathbf{1}$ & Light yellow powder & $55-57$ & $\mathrm{C}_{11} \mathrm{H}_{16} \mathrm{~N}_{2} \mathrm{O}_{2} \mathrm{~S}$ \\
3a & Brownish orange sticky liquid & - & $\mathrm{C}_{13} \mathrm{H}_{20} \mathrm{~N}_{2} \mathrm{O}_{2} \mathrm{~S}$ \\
3b & Brown sticky solid & - & $\mathrm{C}_{14} \mathrm{H}_{20} \mathrm{~N}_{2} \mathrm{O}_{2} \mathrm{~S}$ \\
3c & Mustard sticky solid & - & $\mathrm{C}_{18} \mathrm{H}_{22} \mathrm{~N}_{2} \mathrm{O}_{2} \mathrm{~S}$ \\
3d & Light yellow sticky solid & $\mathrm{C}_{18} \mathrm{H}_{21} \mathrm{BrN}_{2} \mathrm{O}_{2} \mathrm{~S}$ \\
3e & Greenish black sticky solid & $\mathrm{C}_{19} \mathrm{H}_{24} \mathrm{~N}_{2} \mathrm{O}_{2} \mathrm{~S}$ & $\mathrm{C}_{20} \mathrm{H}_{26} \mathrm{~N}_{2} \mathrm{O}_{2} \mathrm{~S}$ \\
3f & Mustard sticky solid & - & $\mathrm{C}_{20} \mathrm{H}_{25} \mathrm{~N}_{3} \mathrm{O}_{3} \mathrm{~S}$ \\
5a & Dark Brown sticky solid & - & $\mathrm{C}_{20} \mathrm{H}_{25} \mathrm{~N}_{3} \mathrm{O}_{4} \mathrm{~S}$ \\
5b & Rust sticky solid & - & $\mathrm{C}_{20} \mathrm{H}_{25} \mathrm{~N}_{3} \mathrm{O}_{4} \mathrm{~S}$ \\
5c & Rust sticky solid & - & 85 \\
\hline
\end{tabular}

TABLE-2

BIOACTIVITY STUDIES OF THE SYNTHESIZED SULFONAMIDES BEARING PIPERIDINE NUCLEUS

\begin{tabular}{|c|c|c|c|c|c|c|}
\hline \multirow[b]{2}{*}{ Compound No. } & \multicolumn{2}{|c|}{$\mathrm{BChE}$} & \multicolumn{2}{|c|}{$\mathrm{AChE}$} & \multicolumn{2}{|c|}{ LOX } \\
\hline & $\begin{array}{c}\text { Inhibition (\%) } \\
\text { at } 0.5 \mathrm{mM}\end{array}$ & $\mathrm{IC}_{50}(\mu \mathrm{M})$ & $\begin{array}{c}\text { Inhibition (\%) } \\
\text { at } 0.5 \mathrm{mM}\end{array}$ & $\mathrm{IC}_{50}(\mu \mathrm{M})$ & $\begin{array}{c}\text { Inhibition (\%) } \\
\text { at } 0.5 \mathrm{mM}\end{array}$ & $\mathrm{IC}_{50}(\mu \mathrm{M})$ \\
\hline 1 & $30.74 \pm 0.42$ & - & $39.49 \pm 0.21$ & - & $57.96 \pm 0.22$ & $329.31 \pm 0.08$ \\
\hline $3 \mathbf{a}$ & $80.67 \pm 0.11$ & $87.51 \pm 0.04$ & $85.98 \pm 0.32$ & $39.21 \pm 0.04$ & $50.06 \pm 0.63$ & $>400$ \\
\hline $3 b$ & $95.68 \pm 0.41$ & $4.4 \pm 0.03$ & $83.90 \pm 0.14$ & $23.21 \pm 0.17$ & $42.17 \pm 0.31$ & - \\
\hline $3 c$ & $95.19 \pm 0.32$ & $4.21 \pm 0.11$ & $75.09 \pm 0.54$ & $60.41 \pm 0.31$ & $48.51 \pm 0.98$ & - \\
\hline 3d & $80.33 \pm 0.31$ & $89.21 \pm 0.014$ & $67.85 \pm 0.25$ & $185.21 \pm 0.39$ & $40.69 \pm 0.34$ & - \\
\hline $3 e$ & $95.53 \pm 0.64$ & $16.21 \pm 0.54$ & $78.69 \pm 0.42$ & $69.71 \pm 0.36$ & $42.95 \pm 0.26$ & - \\
\hline 3f & $94.09 \pm 0.34$ & $17.42 \pm 0.08$ & $82.01 \pm 0.73$ & $147.51 \pm 0.35$ & $49.42 \pm 0.63$ & - \\
\hline $\mathbf{5 a}$ & $96.35 \pm 0.04$ & $18.11 \pm 0.24$ & $86.74 \pm 0.19$ & $32.71 \pm 0.05$ & $67.73 \pm 0.11$ & $339.11 \pm 0.18$ \\
\hline $5 \mathbf{b}$ & $91.47 \pm 0.82$ & $34.41 \pm 0.52$ & $86.46 \pm 0.94$ & $190.52 \pm 0.52$ & $66.11 \pm 0.37$ & $181.31 \pm 0.09$ \\
\hline $5 c$ & $89.79 \pm 0.45$ & $137.24 \pm 0.05$ & $82.01 \pm 0.43$ & $35.31 \pm 0.24$ & $48.90 \pm 0.69$ & - \\
\hline Control & Eserine & $0.85 \pm 0.001$ & Eserine & $0.04 \pm 0.001$ & Baicalein & $22.4 \pm 1.3$ \\
\hline
\end{tabular}

Note: $\mathrm{IC}_{50}$ values (concentration at which there is $50 \%$ enzyme inhibition) of compounds were calculated using EZ-Fit enzyme kinetics software (Perella Scientific Inc. Amherst, USA). LOX = Lipoxygenase. AChE = Acetyl cholinesterase. BChE = Butyryl cholinesterase.

substituted 2-[(phenylsulfonyl)(piperidin-1-yl)amino]acetamide (5a-c), the compound which showed talented inhibitory activity was $N$-(2-methylphenyl)-2-[(phenylsulfonyl)(piperidin-1yl)amino]acetamide (5a) having $\mathrm{IC}_{50}$ value of $18.11 \pm 0.24$ $\mu \mathrm{mol} / \mathrm{L}$ as compared to reference standard. The enhanced activity might be due to the substitution of $N$-(2-methylphenyl)acetamide which is probably more complimentary for the inhibition of butyrylcholinesterase enzyme. The screening against acetylcholinesterase enzyme depicted that the four compounds $\mathbf{3 b}, \mathbf{5 a}, \mathbf{5 c}$ and $\mathbf{3 a}$ exhibited good inhibitory potential but all other synthesized compounds showed moderate activity as compared to relative eserine, a reference standard with $\mathrm{IC}_{50}$ value of $0.04 \pm 0.001 \mu \mathrm{mol} / \mathrm{L}$. However, only few compounds (Table-2) showed very weak inhibition against lipoxygenase enzyme but all other compounds remained inactive.

\section{Conclusion}

The projected structures of the synthesized compounds are well supported by spectroscopic data. The newly synthesized compounds showed varying degree of enzyme inhibition activity.

\section{ACKNOWLEDGEMENTS}

The authors are thankful to Higher Education Commission of Pakistan for financial support.

\section{REFERENCES}

1. F. Sanchez-Sancho and B. Herrandón, Tetrahedron: Asym., 9, 1951 (1998).

2. S. Nithiya, N. Karthik and J. Jayabharathi, Int. J. Pharm. Pharm. Sci., 3, 254 (2011).

3. B. Adger, U. Dyer, G. Hutton and M. Woods, Tetrahedron Lett., 37, 6399 (1996).

4. J.W. Daly and T.F. Spande, In ed.: S.W. Pelletier, Alkaloids: Chemical and Biological Perspectives, Wiley, New York, Vol. 4, pp. 1-254 (1986).

5. A.P. Kozikowski, G.L. Araldi, J. Boja, W.M. Meil, K.M. Johnson and J.L. Flippen, J. Med. Chem., 41, 1962 (1998).

6. M.E. Brau, P. Branitzki, A. Olschewski, W. Vogeland and G. Hempelmann, Anesth. Analg., 91, 1499 (2000).

7. C.T. Supuran, A. Casini and A Scozzafava, Med. Res. Rev., 23, 535 (2003).

8. M. Cygler, J.D. Schrag, J. Sussman, L.M. Harel, I. Silman and M.K., Protein Sci., 2, 366 (1993).

9. V. Tougu, Curr. Med. Chem., 1, 155 (2001).

10. S. Gauthier, Drug Aging, 18, 853 (2001).

11. G. Bertaccini, Handbook of Experimental Pharmacology, Springer Berlin, vol. 59/II, p. 85 (1982).

12. Aziz-ur-Rehman, W. Tanveer, M.A. Abbasi, S. Afroz, K.M. Khan, M. Ashraf and I. Afzal, Int. J. Chem. Res., 3, 99 (2011).

13. G.L. Ellman, K.D. Courtney, V. Andres and R.M. Featherstone, Biol. Pharm., 7, 88 (1961).

14. A.L. Tappel, Arch. Biochem. Biophys., 44, 378 (1953).

15. A.T. Evans, Biol. Pharm., 36, 2035 (1987).

16. S. Baylac and P. Racine, Int. J. Aromatherap., 13, 138 (2003).

17. Aziz-ur-Rehman, S. Afroz, M.A. Abbasi, W. Tanveer, K.M. Khan, M. Ashraf, I. Ahmad, I. Afzal and N. Ambreen, Pak. J. Pharm. Sci., 25, 809 (2012). 